\title{
Pregnancy Outcomes With COVID-19 Lessons Learned From the Pandemic
}

\author{
Saima Siddiqui ${ }^{1}$, Rehana Najam ${ }^{1}$ \\ 1. Department of Obstetrics and Gynaecology, Teerthanker Mahaveer Medical College and Research Centre, \\ Moradabad, IND
}

Corresponding author: Saima Siddiqui, saima.siddiqui03@gmail.com

\section{Abstract \\ Background}

Severe acute respiratory syndrome coronavirus-2 (SARS-CoV-2) that causes Coronavirus disease has caused one of the most damaging pandemics in the recorded human history.

\section{Objective}

To assess pregnancy outcomes with COVID-19 lessons learned from the pandemic.

\section{Study design}

This retrospective observational study was conducted at Teerthanker Mahaveer Medical College and Research Centre, Moradabad, a level 3 COVID hospital in Northern India, with a patient pool of all the antenatal females diagnosed COVID 19 positive via a positive quantitative reverse transcriptase-polymerase chain reaction (qRT-PCR) test of maternal pharyngeal and nasal swab samples in the given time period of three months and ten days, i.e., May 25, 2020 to September 3, 2020. In conjunction with maternal outcomes, neonatal outcomes including evidence of perinatal transmission of SARS-CoV-2 was assessed by testing neonatal pharyngeal swab samples.

\section{Results}

Out of 100 COVID-19 positive patients, the average age of women was 26.2 years, 73 women (73\%) were asymptomatic, and 50 patients (50\%) women had associated co-morbidities such as anaemia in 38 (38\%) women, gestational diabetes mellitus (GDM) and pregnancy-induced hypertension (PIH) in four patients (4\%) each, respectively. No case of spontaneous abortion in early gestation was reported. Out of 100 patients, 32 (32\%) patients delivered during their stay, out of which 17 women (53.1\%) delivered via cesarean section which was performed mainly due to obstetric indications. One maternal death was reported due to antepartum eclampsia which was unrelated to COVID-19 complications. Five neonates were born prematurely, out of which three were delivered followed by spontaneous premature preterm rupture of membranes (PPROM).

Review began $06 / 15 / 2021$ Review ended 07/04/2021 Published 07/13/2021

๑) Copyright 2021 Siddiqui et al. This is an open access article distributed under the terms of the Creative Commons Attribution License CC-BY 4.0., which permits unrestricted use, distribution, and reproduction in any medium, provided the original author and source are credited.
The Appearance Pulse Grimace Activity Respiration (APGAR) score was recorded to be $\geqslant 9$ at the five minutes mark in 28 out of 30 live babies (93.3\%) and the birth weight of the babies ranged from 1.8 to $3.5 \mathrm{~kg}$ ) with an average birth weight of $2.71 \mathrm{~kg}$. Two neonatal deaths were reported due to respiratory distress. There were two documented intrauterine demise (IUD) cases both due to PIH. Furthermore, all 30 live neonates tested for SARS CoV-2 had negative results.

\section{Conclusion}

The spectrum of Coronavirus infection leans more towards asymptomatic and mild symptomatic clinical presentation. Favourably, the likelihood of spontaneous preterm birth was not escalated in our current study and remained low. The rate of intrauterine fetal demise and neonatal death were less. As none of the neonates tested positive for COVID-19, there is no corroborative proof of vertical perinatal transmission.

Categories: Obstetrics/Gynecology, Infectious Disease, Epidemiology/Public Health

Keywords: vertical transmission, neonatal outcome, sars cov-2, covid-19, maternal outcomes, pregnancy outcomes, coronavirus, feto-maternal outcome

\section{Introduction}

Severe acute respiratory syndrome coronavirus-2 (SARS-CoV-2) which causes Coronavirus disease has caused one of the most damaging pandemics in recorded human history [1]. True to its name, the COVID-19 pandemic was first accounted for in December 2019, in Wuhan, China, and the number of positive patients is increasing swiftly across the whole world. As of September 3, 2020, the total confirmed cases of COVID-19 around the world stand at 25,842,652 and have already caused 858,629 deaths all across the world [2].

According to the Indian Council of Medical Research (ICMR), the diagnosis of COVID-19 infection is a fivepillar structure with the pillars being namely, epidemiological vulnerability and exposure, clinical attributes, laboratory parameters, CT chest findings, and a positive qRT-PCR analysis of nasal and pharyngeal 
Pregnancy being a unique physiological and immunological state makes child-bearing females more vulnerable to infections. Due to the increased amount of progesterone and estrogen during pregnancy, the upper respiratory tract becomes inflamed and because of upward shifting of the diaphragm due to the overgrowing uterus, there is also restricted expansion of lungs and therefore all of these factors lead to increased susceptibility to respiratory pathogens. Also, there are concerns owing to the potential results on fetal and neonatal outcomes apart from the consequences of COVID-19 on a child-bearing woman [4].

In 2009, pregnant women constituted only $1 \%$ of all patients infected by the influenza A subtype H1N1 virus, however, they comprised 5\% of the total deaths caused by the H1N1 virus [5]. Likewise, SARS-CoV-2 and Middle Eastern respiratory syndrome (MERS-COV), during their respective outbreaks showed severe complications during pregnancy, leading to admission to intensive care units, endotracheal intubations, renal failure, and ultimately death $[6,7]$.

Considering the impact of previous outbreaks on pregnant women, we realize the pressing need for specialized research and studies to help us figure out the bearing of COVID-19 both short term and long term on a pregnant female as well as her future offspring as the available data are scarce.

\section{Materials And Methods}

This is a retrospective observational study of prospectively collected data of patients admitted in the Department of Obstetrics and Gynaecology at Teerthanker Mahaveer Medical College and Research Center, Moradabad, a level 3 designated COVID hospital in northern India.

According to the Indian Council of Medical Research guidelines, all antenatal women from red-zone/hotspot districts were tested for COVID-19 via reverse transcriptase-polymerase chain reaction (RT-PCR) test even if asymptomatic. The samples were collected either on an outpatient basis or after admission, in accordance with Ministry of Health and Family Welfare, Government of India guidelines.

All the antenatal patients who came out to be COVID-19 positive diagnosed via a positive quantitative qRTPCR test of maternal pharyngeal and nasal swab samples according to ICMR guidelines were included in the study and were admitted at the centre.

The aim and objective of the current study were to assess the effect and outcome of COVID-19 on maternal and neonatal morbidity and mortality. For the aggregation of clinical data such as age, gestational age, parity, address, antenatal history, associated co-morbidities, history of contact/travel, any positive symptoms regarding coronavirus infection were noted in the triage area and the patients were admitted to COVID isolation wards, pre-labor room or to intensive care unit on the basis of their chief complaints and severity of the disease while maintaining all infection prevention and control measures with proper distancing between beds and maintaining proper hand-hygiene and positive sanitary habits. Keeping in mind the infective potential of the female, written and informed consent was relinquished and verbal consent was acquired from the patients and their attendants.

COVID-19 positive patients who were kept in isolation wards and pre-labor rooms were investigated according to the guideline and were prescribed immune-boosters and routine antenatal medications, while symptomatic patients were managed symptomatically in conjunction with the physician and pulmonologist. Asymptomatic patients were discharged according to ICMR guidelines at the time and advised additional strict home quarantine.

After delivery of the baby, the necessary information such as gestational age at the time of delivery, mode of delivery and outcome, cause of cesarean section (if applicable), birth weight of the baby, Appearance Pulse Grimace Activity and Respiration (APGAR) score, need of neonatal intensive care unit (NICU) admission was noted. Neonatal pharyngeal and nasal swabs were sent within 24 hours of birth and the result was taken into consideration. Direct breastfeeding while taking all necessary precautions like wearing an N95 mask, gloves, and hand-washing, and use of sanitizer was actively encouraged in such patients.

Data of 100 COVID-19-positive antenatal women admitted to the hospital within the study period of three months and ten days (May 25, 2020 to September 3, 2020) was retrospectively entered in a Microsoft Excel sheet. Continuous variables are expressed as mean \pm standard deviation (SD). All categorical variables are expressed as frequency and percentages.

\section{Results \\ Demographic data}

Table 1 shows the demographic outlook of the included patients. A maximum number of the patients were in the age group of $20-25$ years. The mean age was $26.17 \pm 3.73$ years; 63 women (63\%) were multigravida. Majority of women presented in their third trimester. Approximately, two-thirds of patients, i.e., 35 antenatal women (35\%) belonged to the upper-middle-class according to the Modified Kuppuswamy scale. 


\section{Cureus}

\begin{tabular}{|c|c|c|}
\hline Parameters & Number of cases $(n)$ & Percentage (\%) \\
\hline \multicolumn{3}{|c|}{ The age group of the patient (years) } \\
\hline$\leq 19$ & 2 & 2 \\
\hline $20-25$ & 47 & 47 \\
\hline $26-30$ & 39 & 39 \\
\hline 31-35 & 10 & 10 \\
\hline$>35$ & 2 & 2 \\
\hline Total & 100 & 100 \\
\hline \multicolumn{3}{|c|}{ Socio-economic data (Modified Kuppuswamy scale) } \\
\hline Upper class (26-29) & 25 & 25 \\
\hline Upper middle (16-25) & 35 & 35 \\
\hline Lower middle (11-15) & 18 & 18 \\
\hline Upper lower (5-10) & 16 & 16 \\
\hline Lower (below 5) & 6 & 6 \\
\hline Total & 100 & 100 \\
\hline \multicolumn{3}{|l|}{ Parity } \\
\hline Primigravida & 37 & 37 \\
\hline Multigravida & 63 & 63 \\
\hline Total & 100 & 100 \\
\hline \multicolumn{3}{|l|}{ Gestational age (weeks) } \\
\hline$>37$ weeks & 38 & 38 \\
\hline 34-37 weeks & 21 & 21 \\
\hline 28-34 weeks & 15 & 15 \\
\hline$<28$ weeks & 26 & 26 \\
\hline Total & 100 & 100 \\
\hline
\end{tabular}

TABLE 1: Demographic Data of the Patients.

\section{Maternal clinical presentation and co-morbidities}

Table 2 shows that 73 women (73\%) were asymptomatic positive without any appearance of symptoms in the period during their stay. Among the 27 symptomatic patients, cough, fever, myalgia, and sore throat were the most common symptoms being $55.5 \%, 37 \%$, 37\%, and $18.5 \%$, respectively. Almost all the patients recovered within two weeks of admission except one patient who required intubation due to antepartum eclampsia. No other patient required oxygen support or ICU admission due to COVID-19 infection. No death was reported due to COVID-19 pneumonia, whereas one patient expired due to an obstetrical cause. As many as $50 \%$ of patients had comorbidities. Anaemia, hypothyroidism, and GDM were the most common comorbidities $38 \%, 10 \%$, and $4 \%$, respectively, as shown in Table 2 . Out of 38 anaemic patients, 11 required blood transfusion. 


\section{Cureus}

\begin{tabular}{|c|c|c|}
\hline Parameters & Cases (n) & Percentage (\%) \\
\hline \multicolumn{3}{|l|}{ Clinical presentation of the patient } \\
\hline Asymptomatic & 73 & 73 \\
\hline Symptomatic & 27 & 27 \\
\hline Total & 100 & 100 \\
\hline \multicolumn{3}{|l|}{ Symptoms n=27 } \\
\hline Fever & 10 & 37 \\
\hline Sore throat & 5 & 18.5 \\
\hline Myalgia & 10 & 37 \\
\hline Diarrhoea & 1 & 3.7 \\
\hline Cough & 15 & 55.5 \\
\hline \multicolumn{3}{|l|}{ Recovery of the patients } \\
\hline Within 15 days & 99 & 99 \\
\hline$>15$ days & 0 & 0 \\
\hline Expired & 1 & 1 \\
\hline \multicolumn{3}{|l|}{ Maternal co-morbidities } \\
\hline DM/GDM & 4 & 4 \\
\hline Hypertension/PIH/eclampsia & 4 & 4 \\
\hline Hypothyroidism & 10 & 10 \\
\hline Anaemia & 38 & 38 \\
\hline Hepatitis C & 1 & 1 \\
\hline
\end{tabular}

TABLE 2: Maternal Clinical Presentation and Co-Morbidities.

DM: diabetes mellitus, GDM: gestational diabetes mellitus, PIH: pregnancy-induced hypertension.

\section{Maternal outcomes}

Out of 100 patients, 32 (32\%) patients got delivered during their stay at the hospital. The number of patients delivered by cesarean section were more, i.e., $53.1 \%$ as compared to vaginal delivery. Cesarean section was done only for an obstetrical indication as shown in Table 3. There were no spontaneous abortions or fetal loss reported. One baby was born before 34 completed weeks via emergency cesarean section in view of previous cesarean section in labour at 33 weeks whereas four babies were born before 37 completed weeks, three vaginal delivery following premature preterm rupture of membrane (PPROM), and one emergency cesarean section due to foetal distress. 


\section{Cureus}

\begin{tabular}{|c|c|c|}
\hline Parameters & Cases (n) & Percentage $(\%)$ \\
\hline \multicolumn{3}{|l|}{ Mode of delivery, $n=32$} \\
\hline Vaginal delivery & 15 & 46.9 \\
\hline Cesarean section & 17 & 53.1 \\
\hline Total & 32 & 100 \\
\hline \multicolumn{3}{|l|}{ Indication of cesarean section, $\mathrm{n}=17$} \\
\hline Previous LSCS in labour & 9 & 52.9 \\
\hline Fetal distress & 5 & 29.5 \\
\hline APH & 1 & 5.8 \\
\hline PIH/eclampsia/HELLP syndrome & 2 & 11.8 \\
\hline COVID-19 pneumonia & 0 & 0 \\
\hline Total & 17 & 100 \\
\hline \multicolumn{3}{|l|}{ Gestational age at delivery } \\
\hline Preterm birth before 34 weeks & 1 & 3.1 \\
\hline Preterm birth before 34 weeks with spontaneous labor-PPROM & 0 & 0 \\
\hline Preterm birth before 37 weeks & 1 & 3.1 \\
\hline Preterm birth before 37 weeks with spontaneous labor-PPROM & 3 & 9.4 \\
\hline Birth after 37 weeks & 27 & 84.4 \\
\hline Total & 32 & 100 \\
\hline
\end{tabular}

\section{TABLE 3: Maternal Outcomes.}

APH: antepartum haemorrhage, PIH: pregnancy-induced hypertension, HELLP syndrome: hemolysis-elevated liver enzymes-low platelet count syndrome, PPROM: premature preterm rupture of membranes, LSCS: lower segment cesarean section.

\section{Neonatal outcomes and complications}

Table 4 shows neonatal outcome and complications in the baby; out of 32 babies delivered, two (6.3\%) were documented intrauterine death (IUD) at the third trimester; the birth weight of the babies ranged from 1.8 to $3.5 \mathrm{~kg}$ with an average birth weight of $2.71 \mathrm{~kg} ; 23$ (71.9\%) babies belonged to a normal weight range of 2.5$3.5 \mathrm{~kg}$, whereas eight (26.7\%) were low-birth-weight, i.e., $<2.5 \mathrm{~kg}$. APGAR score was within normal limits in most of the babies. Low APGAR was observed in two (6.7\%) out of 30 babies (excluding two IUD), five (15.6\%) babies were born preterm and 12 babies (40\%) got admitted to NICU. All the babies tested COVID19 negative implicating that there was no vertical transmission. Two babies expired due to respiratory distress. 


\section{Cureus}

\begin{tabular}{|c|c|c|}
\hline Parameters & Cases (n) & Percentage (\%) \\
\hline \multicolumn{3}{|l|}{ Neonatal outcome } \\
\hline Live term birth & 25 & 78.1 \\
\hline Live preterm birth & 5 & 15.6 \\
\hline IUD & 2 & 6.3 \\
\hline Total & 32 & 100 \\
\hline \multicolumn{3}{|l|}{ Weight at birth $(\mathrm{kg})$} \\
\hline$<2.5 \mathrm{~kg}$ & 8 & 26.7 \\
\hline $2.5-3 \mathrm{~kg}$ & 14 & 46.6 \\
\hline $3.1-3.5 \mathrm{~kg}$ & 8 & 26.7 \\
\hline$>3.5 \mathrm{~kg}$ & 0 & 0 \\
\hline Total & 30 & 100 \\
\hline \multicolumn{3}{|l|}{ APGAR score (At $1 \mathrm{~min}$ ) } \\
\hline $7-10$ & 28 & 93.3 \\
\hline $4-6$ & 2 & 6.7 \\
\hline $0-3$ & 0 & 0 \\
\hline Total & 30 & 100 \\
\hline \multicolumn{3}{|l|}{ Neonatal complications } \\
\hline Baby in NICU & 12 & 40 \\
\hline \multicolumn{3}{|c|}{ Cause of NICU admission, $n=12$} \\
\hline Low birth weight & 9 & 75 \\
\hline Respiratory distress & 2 & 16.7 \\
\hline Meconium stained liquor & 1 & 8.3 \\
\hline Neonatal deaths & 2 & 6.7 \\
\hline COVID 19 positive & 0 & 0 \\
\hline Total live births & 30 & 100 \\
\hline
\end{tabular}

TABLE 4: Neonatal Outcomes and Complications.

IUD: intrauterine death, NICU: neonatal intensive care unit.

\section{Discussion}

Pneumonia is the most ubiquitous non-obstetric infectious condition that transpires during pregnancy. Viral pneumonia has higher rates of maternal as well as neonatal morbidity and mortality as compared to other bacterial etiologies [8]. History has taught us that the aftermath of viral illness in the past like H1N1, SARSCoV, MERS pandemic, and Ebola outbreak has not been great for pregnant females causing various negative obstetrical outcomes including PPROM, preterm labour, intrauterine fetal death, and neonatal death [9-11].

Our current study reported that the majority of women around $74 \%$ acquired infection in their third trimester and belonged to the age group of 20-30 years of age. Studies like Liu et al. [12] and Fan et al. [13] concluded that the majority of patients in their third trimesters acquired infection and the median age was 30 years, similar to our study.

According to the Federation of Obstetrics and Gynaecological Society of India (FOGSI), the majority of people (pregnant and general population) may be asymptomatic or present with mild respiratory symptoms of COVID-19 infection [14]. As reported by the World Health Organisation (WHO), pregnant women having co-morbid conditions such as diabetes, hypertension, obesity, and associated medical risk factors may present with pneumonia and marked hypoxia or may progress rapidly to this state [15]. 
Most of the patients in the current study, around $73 \%$ were asymptomatic, and approximately $50 \%$ of patients had associated co-morbidities as anaemia, hypothyroidism, gestational diabetes mellitus (GDM), and pregnancy-induced hypertension (PIH). The current study reported cough, fever, and myalgia as most common symptoms in the pregnant females in $55.5 \%, 37 \%$, and $37 \%$ of patients, respectively; similar to our study, fever, and cough were reported as the most common clinical manifestation in nine antenatal laboratory-confirmed COVID-19 positive women in their third trimester by Chen et al. [16], whereas sore throat, malaise, myalgia, diarrhoea, and difficulty in breathing were few of the other symptoms reported in the study.

In a study conducted by Nayak et al. [17] on 141 patients, abortion was seen in $4.25 \%$ of patients. In the present study, out of 22 patients presenting in their first and second trimesters, no spontaneous pregnancy loss was reported. In our present study of 100 patients, 32 patients delivered during their stay, out of which $53.1 \%$ delivered via cesarean section. Most studies suggest that most women underwent cesarean section $[13,16,18]$. Recent data tend to incline towards cesarean section; however, the ultimate approach of intrapartum management depends on the woman's co-morbidities.

In the current study, a cesarean section was performed due to obstetric indications like the previous cesarean with scar tenderness, fetal distress, etc., and not because of pulmonary insult caused by COVID-19 pneumonia. While the health of the mother remains a priority, there is another major concern that arises in such cases, that being the impact of SARS CoV-2 infection on the neonate and its vertical transmission potential. In the study by Chen et al. [16], no fetal death, neonatal death, or neonatal asphyxia was reported which was conducted on nine pregnant females. Although there were reports of four neonates being born prematurely, they were not related to the COVID-19 infection. The above result is similar to our current study where five neonates were born prematurely, three followed by spontaneous PPROM.

The APGAR was recorded to be $\geqslant 9$ at the five-minute mark in 28 out of 30 live babies (93.3\%) and $71.9 \%$ of babies have a normal weight between 2.5 and $3.5 \mathrm{~kg}$. Two neonatal deaths were reported due to respiratory distress. Data on the perinatal transmission of SARS CoV-2 infection to the fetus are limited. Many studies like Chen et al. and Zhu et al. have suggested no risk of perinatal transmission [16,19].

In a study by Yan et al. [20], there were $86.0 \%(86 / 100)$ of neonates who undergone testing and their pharyngeal swab test for SARS- CoV- 2 came out to be negative. Of those 86 neonates, 10 newborns had their cord blood samples and amniotic fluid samples tested; 12 and six delivered females had their breast milk and vaginal secretion sample tested, respectively, and the result of all different samples of patients and their newborn came out to be negative.

Thus, the above statements point us towards the conclusion that there is no proof of intrauterine infection via vertical transmission or the risk of perinatal transmission via breastfeeding as similar findings were reported in the current study. There were two documented IUD cases both due to PIH. One maternal death was reported due to antepartum eclampsia which was unrelated to COVID-19 complications.

Our current study has some limitations. The major drawback is the small sample size due to the pressing need in the pool of lacking data. Second, the antenatal patients in their first and second trimester who were discharged after recovering were lost to follow-up and their data leave a void in the research, and follow-up data for post-recovery complications and late neonatal complications are necessary, and therefore, the current study is still ongoing. However, this article is the preliminary window to determine pregnancy outcomes during the COVID-19 pandemic.

\section{Conclusions}

There are only a few studies that deal with the impact and reverberations of SARS CoV-2 infection on childbearing females and their newborns and there is not enough evidence to draw a definitive conclusion on its exact potential. In the current study, the spectrum of Coronavirus infection leans more towards asymptomatic and mild symptomatic clinical presentation. Favourably, the likelihood of spontaneous preterm birth was not escalated in our current study and remained low. The rate of intrauterine Fetal Demise and neonatal death were less. As none of the neonates tested positive for COVID-19, there is no corroborative proof of vertical perinatal transmission and as the sample size is too low, it is difficult to draw a definitive conclusion. However, prolonged follow up is required to keep a check on any latent effects on the newborn. As the number of COVID-19 positive patient continues to increase, more studies are needed to be performed on the pregnant patients and their neonates. As the patient pool of only 100 patients is small, the ongoing aggregation of clinical data and research is currently underway with the aim to answer some pressing questions about the spectrum of the disease, risk of congenital infection, optimal intrapartum approach, and the mode and timing of delivery in order to strengthen the COVID-19 protocols.

\section{Additional Information \\ Disclosures}

Human subjects: Consent was obtained or waived by all participants in this study. IRB-Teerthanker Mahaveer Medical College and Research Centre issued approval IRB/04/2021. With reference to your letter number: OBG/21/81, the IRB has gone through the proposal submitted by you. You are hereby given permission to start your study and inform the IRB about the initiation of the study, provided you do not deviate from the protocol submitted. . Animal subjects: All authors have confirmed that this study did not 
involve animal subjects or tissue. Conflicts of interest: In compliance with the ICMJE uniform disclosure form, all authors declare the following: Payment/services info: All authors have declared that no financial support was received from any organization for the submitted work. Financial relationships: All authors have declared that they have no financial relationships at present or within the previous three years with any organizations that might have an interest in the submitted work. Other relationships: All authors have declared that there are no other relationships or activities that could appear to have influenced the submitted work.

\section{Acknowledgements}

I would like to acknowledge the guidance and contribution of my guide whose undying passion for research and academics has helped me to mould this research article to its present form. I would also like to acknowledge the untiring efforts of our COVID warriors for always motivating me.

\section{References}

1. Hu Y, Sun J, Dai Z, et al.: Prevalence and severity of corona virus disease 2019 (COVID-19): A systematic review and meta-analysis. J Clin Virol. 2020, 127:104371. 10.1016/j.jcv.2020.104371

2. WHO Coronavirus (COVID-19) dashboard. (2021). Accessed: July 13, 2021: https://covid19.who.int.

3. Guidance for management of pregnant women in COVID-19 pandemic . (2020). Accessed: July 13, 2021: https://www.icmr.gov.in/pdf/covid/techdoc/Guidance_for_Management_of_Pregnant_Women_in_COVID19_Pandemic_1204202

4. Liu H, Wang LL, Zhao SJ, Kwak-Kim J, Mor G, Liao AH: Why are pregnant women susceptible to COVID-19? An immunological viewpoint. J Reprod Immunol. 2020, 139:103122. 10.1016/j.jri.2020.103122

5. Siston AM, Rasmussen SA, Honein MA, et al.: Pandemic 2009 influenza A(H1N1) virus illness among pregnant women in the United States. JAMA. 2010, 303:1517-25. 10.1001/jama.2010.479

6. Wong SF, Chow KM, Leung TN, et al.: Pregnancy and perinatal outcomes of women with severe acute respiratory syndrome. Am J Obstet Gynecol. 2004, 191:292-7. 10.1016/j.ajog.2003.11.019

7. Alfaraj SH, Al-Tawfiq JA, Memish ZA: Middle East Respiratory Syndrome Coronavirus (MERS-CoV) infection during pregnancy: report of two cases and review of the literature. J Microbiol Immunol Infect. 2019, 52:501-3. 10.1016/j.jmii.2018.04.005

8. Schwartz DA, Graham AL: Potential maternal and infant outcomes from (Wuhan) Coronavirus 2019-nCOV infecting pregnant women: lessons from SARS, MERS, and other human Coronavirus infections. Viruses. 2020, 12:10.3390/v12020194

9. Maxwell C, McGeer A, Tai KF, Sermer M: Management guidelines for obstetric patients and neonates born to mothers with suspected or probable severe acute respiratory syndrome (SARS). J Obstet Gynaecol Can. 2009, 31:358-64. 10.1016/S1701-2163(16)34155-X

10. Carlson A, Thung SF, Norwitz ER: H1N1 influenza in pregnancy: what all obstetric care providers ought to know. Rev Obstet Gynecol. 2009, 2:139-45.

11. Jamieson DJ, Uyeki TM, Callaghan WM, Meaney-Delman D, Rasmussen SA: What obstetrician-gynecologists should know about Ebola: a perspective from the Centers for Disease Control and Prevention. Obstet Gynecol. 2014, 124:1005-10. 10.1097/AOG.0000000000000533

12. Liu H, Liu F, Li J, Zhang T, Wang D, Lan W: Clinical and CT imaging features of the COVID-19 pneumonia: focus on pregnant women and children. J Infect. 2020, 80:e7-e13. 10.1016/j.jinf.2020.03.007

13. Fan C, Lei D, Fang C, et al.: Perinatal transmission of 2019 Coronavirus disease-associated severe acute respiratory syndrome Coronavirus 2: should we worry?. Clin Infect Dis. 2021, 72:862-4. 10.1093/cid/ciaa226

14. FOGSI GCPR on pregnancy with COVID-19 infection version 2 . (2020). Accessed: July 13, 2021: https://www.fogsi.org/wpcontent/uploads/covid19/fogsi_gcpr_on_pregnancy_with_COVID_19_version_2.pdf.

15. Coronavirus disease 2019 (COVID-19) situation report - 72 . (2019). Accessed: July 13, 2021: https://www.who.int/docs/default-source/coronaviruse/situation-reports/20200401-sitrep-72-covid-19.pdf

16. Chen H, Guo J, Wang C, et al.: Clinical characteristics and intrauterine vertical transmission potential of COVID-19 infection in nine pregnant women: a retrospective review of medical records. Lancet. 2020, 395:809-15. 10.1016/S0140-6736(20)30360-3

17. Nayak AH, Kapote DS, Fonseca M, Chavan N, Mayekar R, Sarmalkar M, Bawa A: Impact of the Coronavirus infection in pregnancy: a preliminary study of 141 patients. J Obstet Gynaecol India. 2020, 70:256-61. 10.1007/s13224-020-01335-3

18. Yu N, Li W, Kang Q, et al.: Clinical features and obstetric and neonatal outcomes of pregnant patients with COVID-19 in Wuhan, China: a retrospective, single-centre, descriptive study. Lancet Infect Dis. 2020, 20:559-64. 10.1016/S1473-3099(20)30176-6

19. Zhu H, Wang L, Fang C, et al.: Clinical analysis of 10 neonates born to mothers with 2019-nCoV pneumonia Transl Pediatr. 2020, 9:51-60. 10.21037/tp.2020.02.06

20. Yan J, Guo J, Fan C, et al.: Coronavirus disease 2019 in pregnant women: a report based on 116 cases . Am J Obstet Gynecol. 2020, 223:111.e1-111.e14. 10.1016/j.ajog.2020.04.014 